\title{
Publisher's Note: Anomalous Hall Effect in a 2D Rashba Ferromagnet [Phys. Rev. Lett. 117, 046601 (2016)]
}

\author{
I. A. Ado, I. A. Dmitriev, P. M. Ostrovsky, and M. Titov
}

(Q) (Received 19 June 2020; published 26 June 2020)

DOI: 10.1103/PhysRevLett.124.259902

This Letter was published online on 20 July 2016 with an omission of a factor in Eqs. 6(a) and 6(b). Equation 6(a) should read as

$$
\sigma_{x y}^{\mathrm{X}}=\frac{1}{2 \pi} \frac{1}{(m \tau)^{2}} \int d^{2} r \operatorname{Tr}\left[J_{r}^{x} G_{-r}^{R} J_{r}^{y} G_{-r}^{A}\right]
$$

and Eq. 6(b) should read as

$$
\sigma_{x y}^{\Psi}=\frac{1}{\pi} \frac{1}{(m \tau)^{2}} \operatorname{Re} \int d^{2} r \operatorname{Tr}\left[J_{r}^{x} G_{-r}^{R} G_{r}^{R} J_{-r}^{y}\right]
$$

The equations have been corrected as of 19 June 2020. The equations are incorrect in the printed version of the journal. 